\title{
Activator Protein-1 Mediates Induced but not Basal Epidermal Growth Factor Receptor Gene Expression
}

\author{
Alfred C. Johnson", Barbara A. Murphy', Christine M. Matelis', \\ Yaffa Rubinstein ${ }^{1}$, Elise C. Piebenga ${ }^{1}$, LaTania M. Akers ${ }^{1}$, Gila Neta ${ }^{1}$, \\ Charles Vinson ${ }^{2}$, and Michael Birrer ${ }^{3}$ \\ ${ }^{1}$ Laboratory of Molecular Biology, ${ }^{2}$ Laboratory of Biochemistry, Division of Basic \\ Sciences and ${ }^{3}$ Biomarkers and Prevention Research Branch, Division of Clinical \\ Sciences, National Cancer Institute, National Institutes of Health, Bethesda, \\ Maryland, U.S.A. \\ Communicated by I. Pastan. Accepted December 2, 1999.
}

\begin{abstract}
Background: The epidermal growth factor receptor (EGFR) is expressed at different levels in many cell types and found overexpressed in many cancers. EGFR expression is increased or decreased in response to extracellular stimuli. We examined the effect of increased $c$-Jun expression on EGFR promoter activity.

Materials and Methods: We used DNAse I footprinting analysis to determine the binding of activator protein 1 (AP-1) to the promoter region. We also used cotransfection experiments and western blotting analysis to determine the effect of AP-1 family members on EGFR expression.

Results: AP-1 was able to bind to at least seven sites in the EGFR promoter region. Cotransfection of MCF-7 cells with a c-Jun expression vector and the EGFR promoter reporter resulted in a 7-fold increase
\end{abstract}

in promoter activity. JunB, but not $c$-fos, also enhanced the EGFR promoter activity. An A-Fosdominant negative shown to inhibit Jun-dependent transactivation was able to prevent c-Jun induction of the promoter activity, but only slightly decreased the basal activity of the promoter. Furthermore, the $A$-Fos dominant negative was able to inhibit phorbol ester induction of the EGFR promoter. Examination of EGFR expression of MCF-7 stable cell lines that overexpress $c$-Jun revealed an increase in EGFR expression. Additionally, a cisplatin-resistant cell line, A2780/CP70, which has an increase in AP-1 activity compared with the parental cell line, A2780, was found to have an increase in EGFR level.

Conclusions: These results indicate that AP-1 can act to increase the expression of EGFR and may play a role in upregulation of EGFR in cancer cells.

\section{Introduction}

Epidermal growth factor (EGF) has a potent mitogenic activity that can either stimulate or inhibit growth of a large variety of normal and malignant cells in vitro $(1,2)$. The ability of EGF

Address correspondence and reprint requests to: Alfred C. Johnson, Ph.D., National Cancer Institute, National Institutes of Health, Building 37 Room 2D 18, 37 Convent Drive MSC-4255, Bethesda, MD 20892-4255. Phone: 301-496-3224; Fax: 301-402-1344; E-mail: aj2e@nih.gov to activate transcription factors in a cell-specific manner and it's signaling activities are mediated through a cell surface receptor, the epidermal growth factor receptor (EGFR) $(3,4)$. The EGFR is a $170 \mathrm{kDa}$ glycoprotein that belongs to the type I growth factor receptor family (5). The cytoplasmic portion of the EGFR is homologous to the viral $\operatorname{erbB}$ oncogene and the EGFR is considered a proto-oncogene (6).

The expression of the EGFR gene is varied in tissues, tumors and cultured cell lines (7). Increasing EGFR levels through retroviral-mediated transfer results in transformation of nor- 
mal cells in the presence of ligand (8-10). Conversely, reducing the level of EGFR can lead to reversion to normal cell phenotype (11). EGFR overexpression has been correlated with poor prognosis and failure to respond to hormonal treatment in breast cancer and in malignant tissue in general (12-14). The EGFR is considered a marker for cell transformation $(15,16)$, making it an attractive target for clinical intervention.

The regulatory region of the EGFR gene has been isolated and characterized $(17,18)$. It lacks the classical TATA or CAAT boxes, but contains multiple transcriptional initiation sites and five GC boxes $(17,19,20)$. The EGFR promoter contains binding sites for transcription factors such as Spl $(19,21)$, AP2 (22), p53 (23-25), WT1 (26), and GCF (27-29). Recent studies show that the promyelocytic leukemia (PML) protein, a nuclear phosphoprotein that functions as a transcriptional regulator, interacts with Spl and inhibits its transactivation of the EGFR promoter (30-32). The PML gene is located at the breakpoint of the $t(15,17)$ translocation in acute promyelocytic leukemia $(33,34)$.

The activator protein-1 (AP-1) transcription factors are immediate, early-response genes involved in a variety of regulatory processes including cellular response to growth factor stimuli (35). AP- 1 is a dimer formed by one protein of the jun family (c-Jun, JunB, JunD) (36-39) and one member of the fos family (c-fos, FosB, Fra-1 and Fra-2) (40-43). AP-1 interacts with a nucleotide sequence motif known as the phorbol 12-O tetradecanoylphorbol 13 acetate (TPA) response element (TRE) (TGAC/GTCA) located in the regulatory region of responsive genes, many of which are involved in cell growth, differentiation and transformation. AP-1 can also bind to the cAMP response element (CRE) with a lower affinity $(44,45)$. Jun proteins can homodimerize with themselves or heterodimerize with Fos proteins. The regions of Jun that are necessary for transcriptional activation include the DNA-binding domain, the leucine zipper and the transcriptional activation domain.

APl transcription factor activity has been shown to be lower in breast cancer cell lines than normal mammary epithelial cells (46). Breast cancer cell lines can possess as few as 2000 EGFR/cell and as many as two million (14). The variability of EGFR levels in breast cancer cell lines prompted us to examine whether APl could regulate EGFR expression. We showed previously that AP2 could bind to the EGFR promoter and partially mediate phorbol ester induction of promoter activity (22). In this study, we determined whether API could bind to the EGFR promoter and examined the effect of c-Jun overexpression on EGFR promoter activity. We also determined the effect of a dominant negative to API that abolishes DNA binding and inhibits oncogenesis. Furthermore, we analyzed the effect of overexpression of c-Jun on EGFR levels in MCF-7 cells. The results from this study indicate that APl is involved in regulating EGFR levels and participates in the phorbol ester enhancement of EGFR gene expression.

\section{Materials and Methods}

Cell Lines and Culture Conditions

NIH3T3 cells were grown in Dulbecco's modified Eagle's medium (DMEM) media supplemented with $10 \%(\mathrm{v} / \mathrm{v})$ calf serum, $2 \mathrm{mM}$ glutamine and penicillin/streptomycin (Life Technologies, Inc., Gaithersburg, MD). The human breast cancer cell line MCF-7 was grown in RPMI-1640 media supplemented with $10 \%$ (v/v) fetal calf serum, $2 \mathrm{mM}$ glutamine and penicillin/streptomycin. The c-Jun-overexpressing cell lines, 2-16, 2-31, 2-33 and the control neomycin-resistant cell lines, 7-1 and 7-2 were maintained in improved minimal Eagle's medium (IMEM) zinc optional media supplemented with $10 \%(\mathrm{v} / \mathrm{v})$ fetal calf serum, $2 \mathrm{mM}$ glutamine, penicillin/streptomycin and $500 \mu \mathrm{g} / \mathrm{ml} \mathrm{G418}$. Human epidermoid carcinoma KB cells and human WI-38 cells were grown in DMEM media supplemented with $10 \%(\mathrm{v} / \mathrm{v})$ fetal calf serum, $2 \mathrm{mM}$ glutamine and penicillin/streptomycin. The human ovarian cell line A2780 and the cisplatin-resistant cell line A2780/CP70 were maintained in RPMI-1640 media supplemented with $2 \mathrm{mM}$ glutamine, 0.2 units $/ \mathrm{ml}$ insulin and penicillin/streptomycin.

\section{Luciferase Assays}

NIH3T3, MCF-7 and KB cells grown in triplicate in $35 \mathrm{~mm}$ diameter plate were transfected with the appropriate expression vector by lipofectamine (Life Technologies, Gaithersburg, MD). Luciferase reporter constructs containing the EGFR promoter were prepared by ligation 
of the HinD III promoter fragments from EGFR-CAT constructs into pGL3-Basic (Promega, Madison, WI). The EGFR promoter reporter plasmids $(0.1 \mu \mathrm{g})$ were cotransfected with the indicated amount of expression vectors and with the control vectors. DNA concentration was kept constant by addition of herring sperm DNA. Cells were harvested $24 \mathrm{~h}$ after transfection and cell extracts prepared according to the protocol from Analytical Luminescence Laboratory, San Diego, CA. All luciferase activities were normalized for protein concentration and transfection efficiency using RSV- $\beta$-galactosidase. All experiments were performed in triplicate or more.

\section{DNase I Footprinting}

DNase I footprinting was performed according to Dynan et al. (47). The EGFR gene promoter fragment $(-1109$ to -16$)$ was labeled at the HindIII site and a 718 base pair $(-734$ to -16$)$ fragment and a 375 base pair ( -1109 to -734 ) fragment isolated after cutting with Pst I. Purified AP1, AP2, TFIID and Spl were purchased from Promega Corporation.

\section{Western Blots}

Whole cell extracts were prepared from cells grown until $80-90 \%$ confluent in the appropriate medium. Cells were washed three times with phosphate buffered saline without calcium and magnesium. Cells were harvested and resuspended in extraction buffer containing 20 mM N-2-hydroxyethylpiperazine-N-2-ethanesulfonic acid (HEPES) 7.9, $0.2 \%$ NP40, $10 \%$ glycerol, $0.1 \mathrm{mM}$ EDTA, $0.4 \mathrm{M} \mathrm{NaCl}, 1 \mathrm{mM}$ DTT and protease inhibitors. Proteins were extracted while rotating at $4^{\circ} \mathrm{C}$ for $30 \mathrm{~min}$. After extraction, samples were centrifuged at 14,000 $\mathrm{g}$ for $15 \mathrm{~min}$. Supernatants were collected and stored at $-80^{\circ} \mathrm{C} .50 \mu \mathrm{g}$ of protein from whole cell extracts were resolved on a 4-12\% SDSpolyacrylamide gel, transferred onto PVDF membrane (Novex, Carlsbad, CA), followed by immunoblotting with the required antibodies, using the ECL detection system according to the manufacturer instructions (Amersham, Pharmacia Biotech, Uppsala, Sweden). PVDF membranes were stripped and probed with actin antibodies. Antibodies to EGFR were purchased from Santa Cruz Biotechnology (Santa Cruz, CA) and actin antibodies were purchased from Boehringer-Mannheim (Mannheim, Germany).

\section{Results}

We showed previously that EGFR expression was enhanced by phorbol esters, in part, through increased binding of AP2, a member of the helix-loop-helix family of transcription factors (22). To extend these analyses, we investigated whether API could also enhance EGFR promoter activity. Examination of the $1.1 \mathrm{kbp}$ EGFR promoter sequence revealed 11 potential AP1 binding sites (TKAGTCA) that differed from the consensus by one or two nucleotides. Most of the potential sites were located in the 5 ' portion of the promoter, but three were located in the $3^{\prime}$ proximal region that was shown to be sufficient for full promoter activity. To determine if APl could bind to the promoter region, we performed DNase I footprinting analysis. Using two fragments of the promoter, -1109 to -734 and -734 to -16 , we detected at least seven protected regions due to API binding (Fig. 1). Four of the seven footprints corresponded to potential recognition sequences, but three did not. Some of the footprints overlapped binding sites for AP2, Spl and TFIID (Fig. 2). The recognition sequences and nucleotide positions of the AP1 footprints are summarized in Table 1.

To examine whether APl could influence EGFR promoter activity, we cotransfected MCF7 cells with a $c$-Jun expression plasmid and the EGFR promoter reporter plasmid, pERl-Luc. The activity of the EGFR reporter construct was increased in a concentration dependent manner to approximately 7 -fold by c-Jun cotransfection (Fig. 3). As a positive control, an API-responsive collagenase promoter construct, API-Col-Luc,

Table 1. Summary of EGFR promoter API footprints

\begin{tabular}{|c|c|c|c|}
\hline \multirow{2}{*}{$\frac{\text { Footprint }}{1}$} & \multicolumn{2}{|c|}{ Position } & \multirow{2}{*}{$\begin{array}{c}\begin{array}{c}\text { Recognition } \\
\text { Sequence }\end{array} \\
\text { CTGACTCC }\end{array}$} \\
\hline & -54 to & -44 & \\
\hline 2 & -102 to & -92 & CCGAGTCC \\
\hline 3 & -170 to & -160 & CGGAGTCC \\
\hline 4 & -760 to & -745 & TCTGATCC \\
\hline 5 & -835 to & -819 & TTAGAGGC \\
\hline 6 & -858 to & -872 & TTGACAAG \\
\hline 7 & -1028 to & -1010 & TTGTGTCA \\
\hline
\end{tabular}


A.

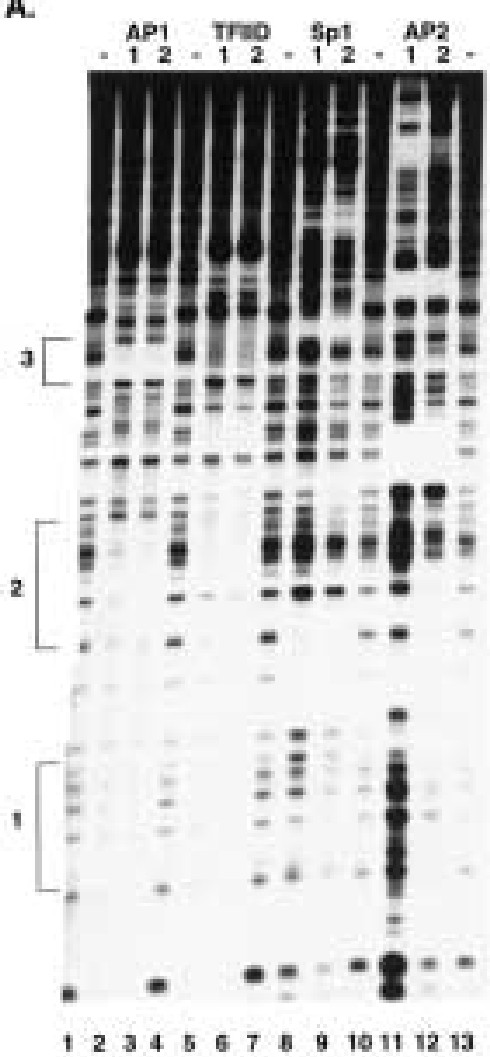

B.

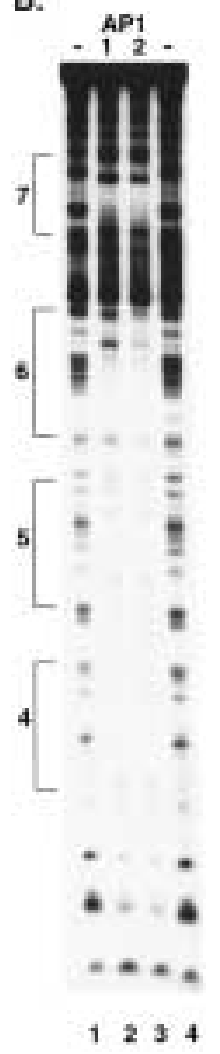

Fig. 1. APl can bind to multiple sites in the EGFR promoter. (A) DNase I footprinting was performed using 1 and 2 footprint units of purified proteins (AP1, TFIID, Spl and AP2 as shown above each lane) and the end-labeled EGFR promoter fragment -16 to -734 . APl footprints are indicated on the left of the figure. (B) DNase I footprinting was performed using 1 and 2 footprint units of API and the EGFR promoter fragment -1109 to -735 . APl footprints are indicated on the left of the figure.

was used and enhanced 8- to 10-fold by c-Jun. As a negative control, the mutant construct, mAPl-Col-Luc, containing a mutation in the TRE was used and not enhanced by c-Jun. Similar results were obtained in mouse NIH3T3 cells and human $\mathrm{KB}$ cells in addition to the MCF-7 cells. To locate the region(s) in the promoter responsive to $c$-Jun cotransfection, experiments with deletion mutants of the EGFR promoter were performed. These experiments revealed that the promoter deletions down to nucleotide -105 were responsive to $c$-Jun (Fig. 4). The deletion constructs containing the most APl sites were more responsive than the ones with fewer sites. To further investigate the c-Jun enhancement of EGFR promoter activity, we cotransfected MCF-7 cells with pERI-Luc and

other Jun and Fos constructs. A c-Jun deletion mutant lacking the transactivation domain, $c$ Juns 1-286, failed to enhance EGFR promoter activity (Fig. 5). Another member of the c-Jun family, JunB, was able to increase EGFR promoter activity an average of 12 -fold, but a $c-f o s$ construct did not successfully enhance the promoter activity.

To further substantiate the role of c-Jun in the activation of EGFR promoter activity, we decided to use a dominant negative construct that was based on a unique structural element. The $A$-Fos dominant negative contains an amphipathic acidic extension appended to the Fos leucine zipper motif that inhibits c-Jun dependent transcription (48). Cotransfection of the $A$-Fos dominant negative and the EGFR promoter reporter resulted in a $30 \%$ decrease in promoter activity (Fig. 6). The A-Fos dominant negative was significantly more effective at inhibiting the activity of APl-responsive constructs, TRE2x-Luc and API-Col-Luc, 80 and $90 \%$, respectively. This result suggests that basal EGFR promoter activity is only slightly due to an effect of API.

To further investigate the role of API in regulating EGFR promoter activity, we treated cells cotransfected with $A$-Fos and the EGFR reporter with phorbol 12-myristate 13-acetate (PMA). PMA increased promoter activity as we had previously reported (Fig. 7). However, $A$ Fos was able to inhibit the PMA induction of the promoter activity. In addition, we cotransfected cells with c-Jun and A-Fos to see if A-Fos could specifically inhibit $c$-Jun activation of EGFR promoter activity. A-Fos reduced $c$-Jun activation to 2-fold, compared with 7-fold in the absence of $A$-Fos. A dominant negative to the CAAT/enhancer binding protein (C/EBP), A$\mathrm{C} / \mathrm{EBP}$, was used as a control. A-C/EBP has the same amphipathic acidic extension as $A$-Fos appended to the C/EBP leucine zipper (48). AC/EBP failed to inhibit $c$-jun-dependent activation of the EGFR promoter. These results indicate that APl plays a role in activation of the EGFR gene by phorbol esters.

The RNA and protein levels of the EGFR gene are varied in many types of cells. To determine if the EGFR level may change in response to APl activity, we examined the level of the receptor in a parental cell line, A2780, and cis-platin resistant cell line, A2780/CP70, that has higher APl activity $(49,50)$. Western blots analysis showed that EGFR level increased in the A2780/CP70 cells, compared 


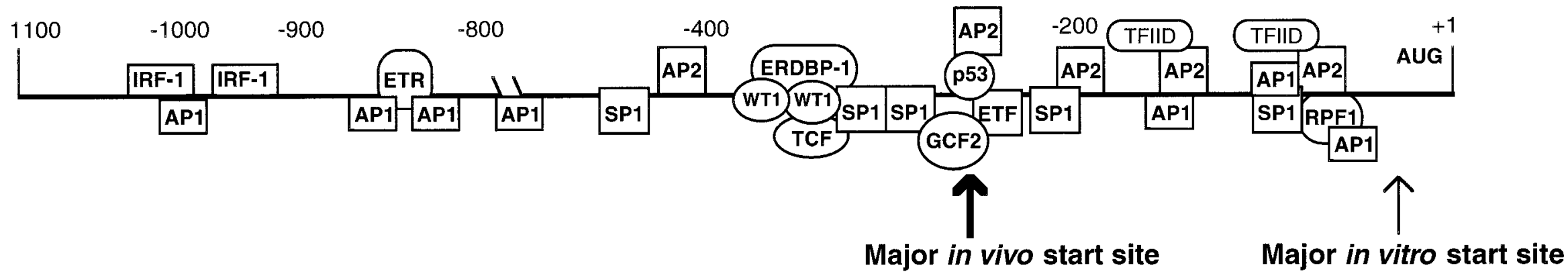

Fig. 2. EGFR promoter schematic with transcription factor binding sites. The schematic of the $1.1 \mathrm{kbp}$ EGFR promoter region with currently known protein binding sites. The size of the symbols for each protein does not correspond to size. The positions of the binding sites on the promoter fragment are representative of loca- tion, if not exact. References for each protein binding: APl (this paper), AP2 (22), ERDBP-1 (56), ETF (21), ETR (57), GCF2 (58), IRF-1 (53), p53 (24), RPF-1 (59), Spl (19), TCF (52), TFIID (this paper), WT1 (26). 


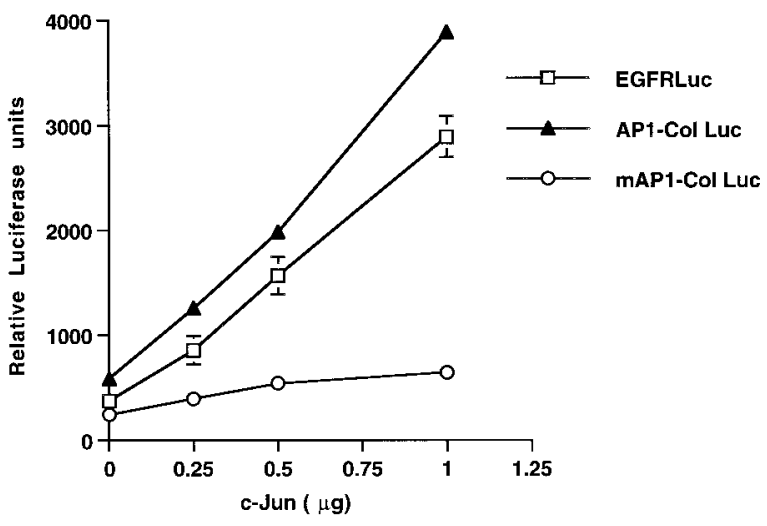

Fig. 3. EGFR promoter activity is increased by $c$-Jun in cotransfection assays. MCF-7 cells were transfected with $0.1 \mu \mathrm{g}$ of reporter construct and increasing amounts of $c$-Jun. Luciferase activity was measured $24 \mathrm{hr}$ post-transfection and is expressed relative to cells transfected with the empty vector. Luciferase activity was corrected for protein concentration and transfection efficiency using a RSV$\beta$-galactosidase reporter.

with the A2780 (Fig. 8). To more thoroughly determine if increased APl activity could influence EGFR levels, we examined the level of the EGFR in MCF-7 stable cell lines that overexpressed c-Jun (51). As shown in Figure 9, cell lines that stably expressed c-Jun have an increase in EGFR level. This is in agreement with the transient cotransfection experiments that showed increase EGFR promoter activity by c-Jun.

\section{Discussion}

We report here that APl plays a role in the regulation of EGFR gene expression. This finding is based on the observation that API binds to at least seven sites in the EGFR promoter region. Also, c-Jun increases EGFR promoter activity in a concentration-dependent manner. Furthermore, an A-Fos-dominant negative that abolishes AP1 DNA-binding inhibits both c-Jun and phorbol ester activation of the EGFR. Finally, cells made to stably overexpress $\mathrm{c}$-Jun have increased EGFR levels. The activation of EGFR promoter activity by $c$-Jun requires the transactivation domain. The effects of c-Jun are spread throughout the promoter region where APl binding sites are located. The API binding sites include sites that overlap with binding sites for Sp1, AP2 and TFIID (Fig. 2). This indicates that the interplay of transcription fac-

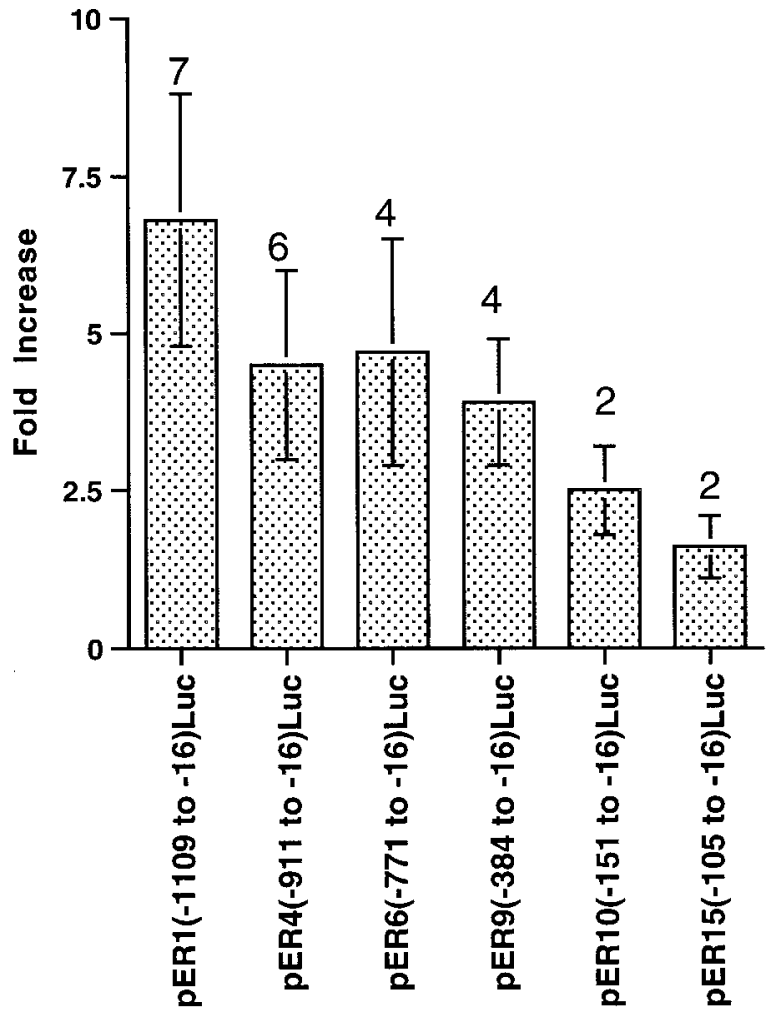

Fig. 4. Enhancement of EGFR promoter activity by $c$-Jun is not localized to a single region of the promoter. MCF-7 cells were transfected with $0.1 \mu \mathrm{g}$ EGFR promoter deletion constructs and $1 \mu \mathrm{g}$ of the c-Jun expression vector. Luciferase activity was measured $24 \mathrm{hr}$ post-transfection and is expressed relative to cells transfected with the empty vector. Luciferase activity was corrected for protein concentration and transfection efficiency using a $\mathrm{RSV}-\beta$-galactosidase reporter. The number of API binding sites in each promoter construct is shown above the error bars.

tors could be extremely important for EGFR gene expression. Over-expression of $c$-Jun leads to increased levels of EGFR. It is also significant that an ovarian cancer cell line selected for cisplatin resistance has increased EGFR levels, as well as increased APl activity. Since cells with higher levels of EGFR are more responsive to the mitogenic action of growth factors, the increase in EGFRs may provide an additional growth advantage for cancer cells that are resistant to chemotherapeutic agents.

The EGFR promoter is responsive to many agents and regulation has to be properly maintained to insure proper cell growth. Transcriptional activators such as Sp1, AP2, p53, TCF and IRF-1 have been shown to bind to the promoter region and enhance transcription $(19,21,22,24,52,53)$. The effect of Spl can 


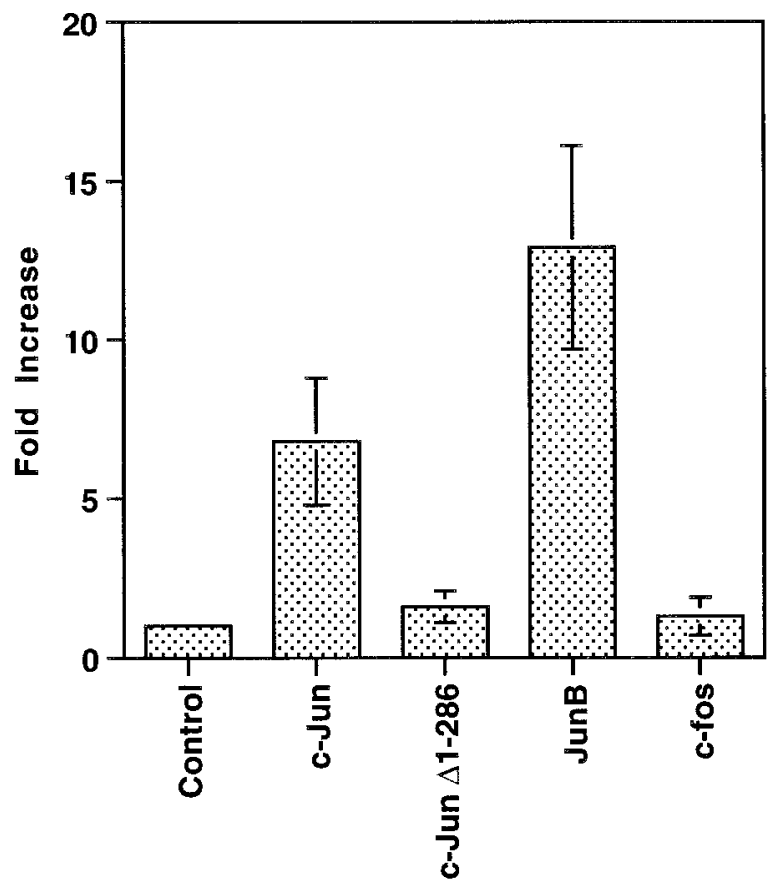

Fig. 5. c-Jun and JunB, but not $c$-fos, in EGFR promoter activity. MCF-7 cells were transfected with $0.1 \mu \mathrm{g}$ EGFR pER l-luc and $1 \mu \mathrm{g}$ of the indicated expression vectors. Luciferase activity was measured $24 \mathrm{hr}$ post-transfection and is expressed relative to cells transfected with the control (empty vector). Luciferase activity was corrected for protein concentration and transfection efficiency using a RSV- $\beta$-galactosidase reporter.

be inhibited by interactions with PML. The repressive effect of PML on EGFR gene transcription was mapped to the region between -150 and -16 . The primary effect of $c$-Jun appears to involve two areas of activity, -150 to -44 and -1028 to -745 . The -1028 to -745 region contains four APl binding sites. Indeed, placing a region of the EGFR promoter containing nucleotides -1109 to -569 upstream of a thymidine kinase (tk) minimal promoter makes the tk promoter responsive to $\mathrm{c}$-Jun activation (data not shown). The -170 to -44 region of the promoter contains three APl binding sites and $60 \%$ of the induced activity by c-Jun. This region of the promoter is very important for in vitro and in vivo activities. Inhibition of $c$-Jun activation by the A-Fos-dominant negative allows for two conclusions: 1.) The effect of c-Jun is primarily an induction of the promoter activity. A-Fos was only able to slightly decrease the basal activity. 2.) The enhancement of EGFR expression by phorbol esters is, at least,

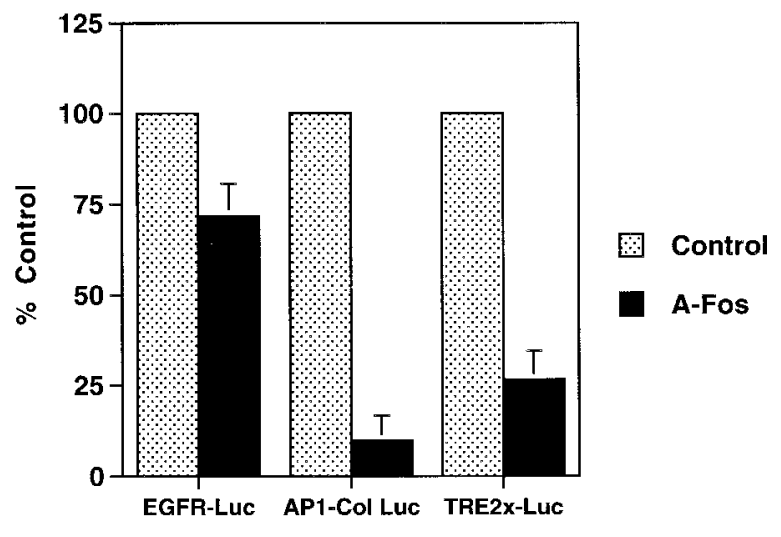

Fig. 6. An A-Fos dominant-negative only slightly decreases EGFR promoter basal activity. MCF-7 cells were transfected with $0.1 \mu \mathrm{g}$ pER 1Luc, APl-Col-Luc or TRE2x-luc and $1 \mu \mathrm{g}$ of the $A$-Fos expression vector. Luciferase activity was measured $24 \mathrm{hr}$ post-transfection and is expressed relative to cells transfected with the empty vector. Luciferase activity was measured 24 hr posttransfection and is expressed as fold increase relative to cells transfected with the empty vector. Luciferase activity was corrected for protein concentration and transfection efficiency using a RSV- $\beta$-galactosidase reporter. The reported data is from three experiments performed in triplicates.

in part, due to increased APl activity. The A-Fos-dominant negative was able to almost completely inhibit the phorbol ester induction of EGFR promoter activity. Previously, we showed that AP2 was able to mediate phorbol ester induction of EGFR gene expression. In that report, we were able to partially purify a protein whose presence in the extract correlated with increased binding to the promoter upon PMA treatment. The DNase I footprint of this factor on the EGFR promoter region matched AP2, except for two protected regions. These sites matched API binding sites (data not shown). In vitro transcription assays were used to show that addition of AP2 leads to an increase in EGFR promoter transcription. Taken together, the results from the previous report and the present study suggest that both AP1 and AP2 play roles in mediating the phorbol ester enhancement of EGFR gene expression.

The role of other members of the APl family in regulating EGFR activity remains to be examined. We showed that JunB was able to increase EGFR promoter activity in the cotransfection assays. Conversely, c-fos addition was not able to enhance EGFR promoter activ- 


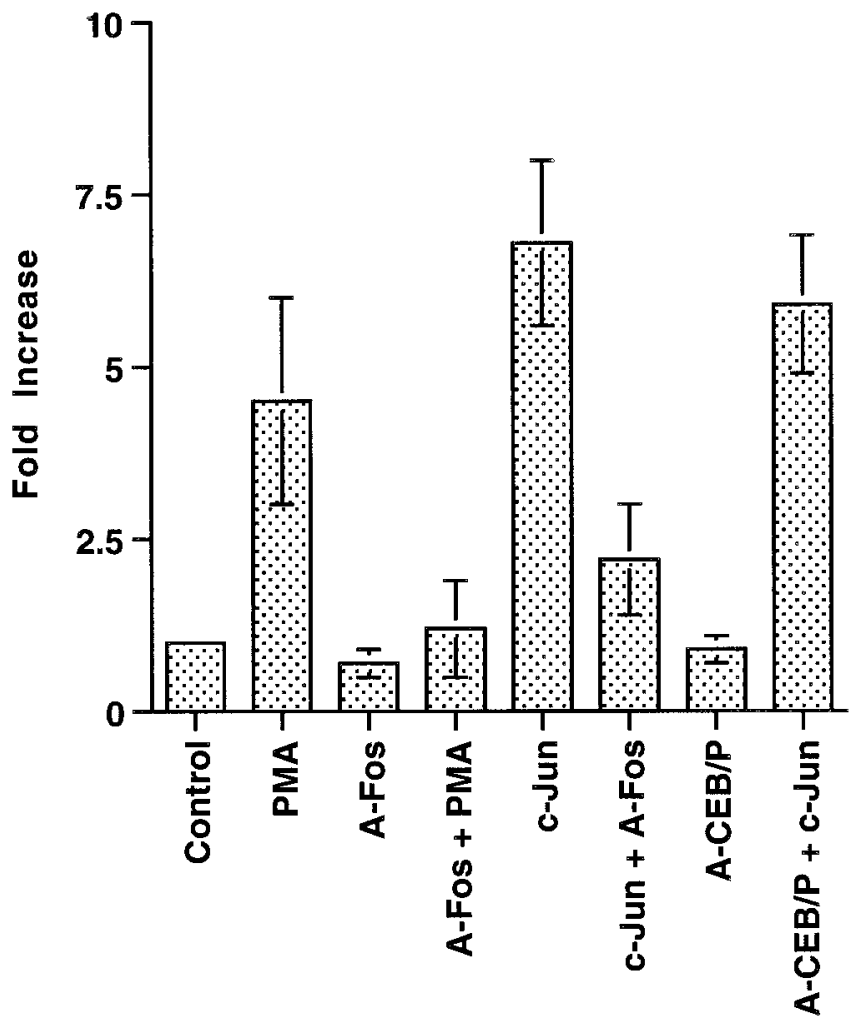

Fig. 7. An A-Fos dominant negative inhibits PMA and $c$ Jun activation of the EGFR promoter. MCF-7 cells were transfected with $0.1 \mu \mathrm{g}$ pER I-Luc and treated for $19 \mathrm{hr}$ with PMA (100 $\mathrm{nM}$ ) before harvesting. In cotransfection experiments, $A$-Fos, A$\mathrm{CEB} / \mathrm{P}$ and/or $\mathrm{c}$-Jun expression vectors $(1.0 \mu \mathrm{g})$ were also transfected. Luciferase activity was measured $24 \mathrm{hr}$ post-transfection and is expressed relative to cells transfected with the empty vector. Luciferase activity was corrected for protein concentration and transfection efficiency using a RSV- $\beta$-galactosidase reporter. ity. These findings indicate that endogenous levels of specific factors play an important role in determining EGFR promoter activity in transient transfection assays. Indeed, we find very low levels of c-Jun and JunB in our MCF-7 cells
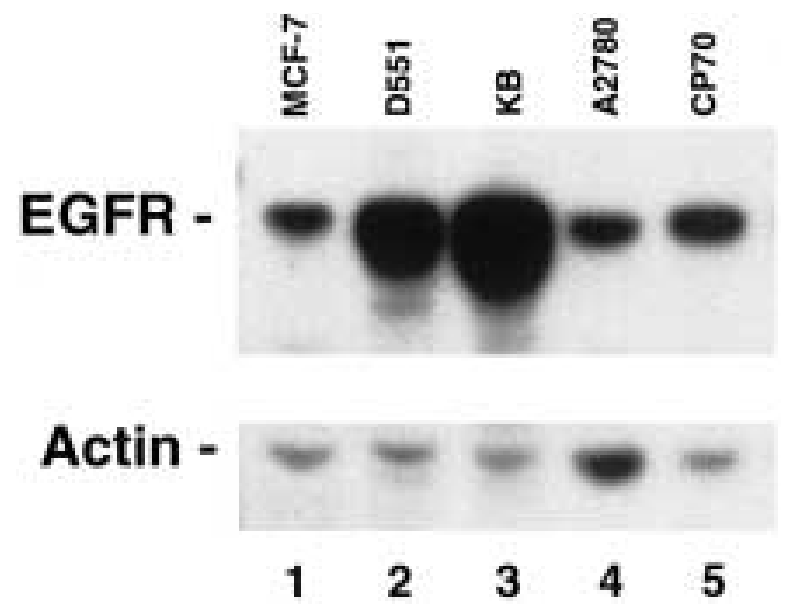

Fig. 8. EGFR is increased in cis-platin resistant cells that have increased API activity. Protein extracts from MCF-7, D551, KB, A2780 and A2780/CP70 cells were as described in "Materials and Methods." $50 \mu \mathrm{g}$ of each extract was fractionated on polyacrylamide gels and subjected to Western blot analysis using EGFR and actin antibodies. by western blotting (data not shown). These findings also indicate that transcription factors involved in activation of specific gene expression may not have a major role in basal expression. The identification of the additional tran-

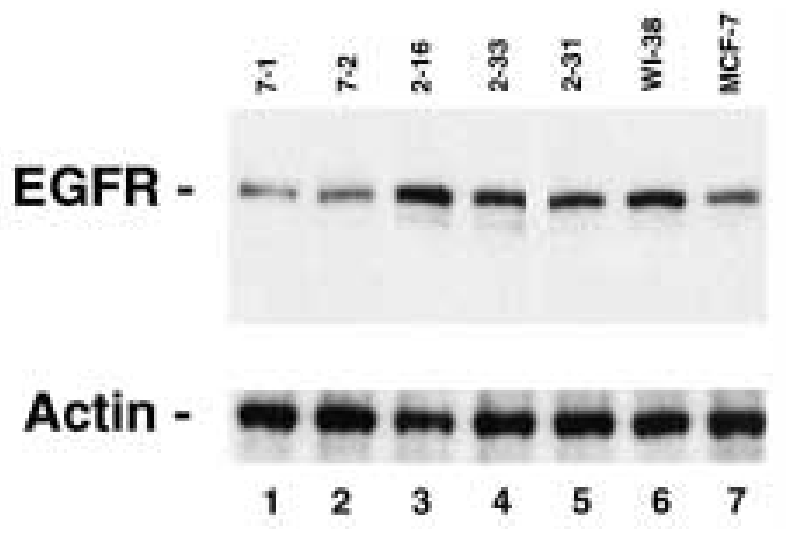

Fig. 9. Endogenous EGFR is increased in MCF-7 cells over-expressing $c$-Jun. Stable transfectants of MCF-7 expressing neomycin resistance (7-1 and 72 ) or c-jun (2-16, 2-33 and 2-31) were grown in selective media and protein extracts prepared. Extracts from MCF-7 and WI-38 cells were prepared in an identical fashion. $50 \mu \mathrm{g}$ of each extract was fractionated on polyacrylamide gels and subjected to western blotting using EGFR and actin antibodies. 
scription factors effecting changes in EGFR expression will aid in resolving the increased expression found in many cancers.

It is clear that EGFR gene regulation is very complex and involves the interaction of many different transcription factors with the promoter region $(22,54)$. The EGFR gene responds to growth factors, tumor-promoting agents, cAMP, steroids, and retinoids (54). These agents induce the expression of transcription factors such as API (35). The APl complex can either positively or negatively regulate transcription of a target gene, depending on the composition of the heterodimers. Some breast cancer cells have been shown to have lower AP1 activity than normal mammary epithelial cells (46). APl has also been implicated in the regulation of apoptosis by either inducing apoptosis or inhibiting anti-apoptotic events (55). The role of API in regulating EGFR expression adds support for a role in regulating cell proliferation.

\section{References}

1. Carpenter G. (1990) Epidermal growth factor. J. Biol. Chem. 265: 7709-7712.

2. Dittadi R, Donisi PM, Brazzale A, Cappellozza L, Bruscagnin G, Gion M. (1993) Epidermal growth factor receptor in breast cancer. Comparison with nonmalignant breast tissue. Br. J. Cancer 67: 7-9.

3. David M, et al. (1996) STAT activation by epidermal growth factor (EGF) and amphiregulin. Requirement for the EGF receptor kinase but not for tyrosine phosphorylation sites or JAK I. J. Biol. Chem. 271: 9185-9188.

4. Merlino GT. (1990) Epidermal growth factor receptor regulation and function. Semin. Cancer Biol. 1: 277-284.

5. Rajkumar T, Gullick WJ. (1994) The type I growth factor receptors in human breast cancer. Breast Cancer Res. Treat. 29: 3-9.

6. Downward J, et al. (1984) Close similarity of epidermal growth factor receptor and v-erb-B oncogene protein sequences. Nature 307: $521-$ 527.

7. King, CR, et al. (1985) Human tumor cell lines with EGF receptor gene amplification in the absence of aberrant sized mRNAs. Nucleic Acids Res. 13: 8477-8486.

8. Velu TJ, et al. (1987) Epidermal-growth-factordependent transformation by a human EGF receptor proto-oncogene. Science 238: 14081410.

9. Velu TJ, Beguinot L, Vass WC, Zhang K, Pastan I, Lowy DR. (1989) Retroviruses expressing dif- ferent levels of the normal epidermal growth factor receptor: biological properties and new bioassay. J. Cell Biochem. 39: 153-166.

10. Di Fiore PP, et al. (1987) Overexpression of the human EGF receptor confers an EGF-dependent transformed phenotype to NIH $3 \mathrm{~T} 3$ cells. Cell 51: 1063-1070.

11. Moroni MC, et al. (1992) EGF-R antisense RNA blocks expression of the epidermal growth factor receptor and suppresses the transforming phenotype of a human carcinoma cell line. J. Biol. Chem. 267: 2714-2722.

12. LeMaistre CF, Meneghetti C, Howes L, Osborne CK. (1994) Targeting the EGF receptor in breast cancer treatment. Breast Cancer Res. Treat. 32: 97103.

13. Nicholson RI, et al. (1994) Epidermal growth factor receptor expression in breast cancer: association with response to endocrine therapy. Breast Cancer Res. Treat. 29: 117-125.

14. Chrysogelos SA, Dickson RB. (1994) EGF receptor expression, regulation, and function in breast cancer. Breast Cancer Res. Treat. 29: 29-40.

15. Budillon A, et al. (1991) Upregulation of epidermal growth factor receptor induced by alphainterferon in human epidermoid cancer cells. Cancer Res. 51: 1294-1299.

16. Iacopino F, Ferrandina G, Scambia G, BenedettiPanici P, Mancuso S, Sica G. (1996) Interferons inhibit EGF-stimulated cell growth and reduce EGF binding in human breast cancer cells. Anticancer Res. 16: 1919-1924.

17. Ishii S, Xu YH, Stratton RH, Roe BA, Merlino GT, Pastan I. (1985) Characterization and sequence of the promoter region of the human epidermal growth factor receptor gene. Proc. Natl. Acad. Sci. U.S.A. 82: 4920-4924.

18. Haley J, Whittle N, Bennet P, Kinchington D, Ullrich A, Waterfield M. (1987) The human EGF receptor gene: structure of the $110 \mathrm{~kb}$ locus and identification of sequences regulating its transcription. Oncogene Res. 1: 375-396.

19. Johnson AC, et al. (1988) Epidermal growth factor receptor gene promoter. Deletion analysis and identification of nuclear protein binding sites. J. Biol. Chem. 263: 5693-5699.

20. Kageyama R, Merlino GT. (1991) In vitro transcription of epidermal growth factor receptor gene. Methods Enzymol. 198: 242-250.

21. Kageyama R, Merlino GT, Pastan I. (1988) Epidermal growth factor (EGF) receptor gene transcription. Requirement for $\mathrm{Spl}$ and an EGF receptor-specific factor. J. Biol. Chem. 263: 63296336.

22. Johnson AC. (1996) Activation of epidermal growth factor receptor gene transcription by phorbol 12-myristate 13-acetate is mediated by activator protein 2. J. Biol. Chem. 271: 3033-3038.

23. Deb SP, Munoz RM, Brown DR, Subler MA, Deb S. (1994) Wild-type human p53 activates 
the human epidermal growth factor receptor promoter. Oncogene. 9: 1341-1349.

24. Ludes-Meyers JH, et al. (1996) Transcriptional activation of the human epidermal growth factor receptor promoter by human p53. Mol. Cell Biol. 16: 6009-6019.

25. Sheikh MS, et al. (1997) Identification of an additional p53-responsive site in the human epidermal growth factor receptor gene promotor. Oncogene 15: 1095-1101.

26. Englert C, et al. (1995) WT1 suppresses synthesis of the epidermal growth factor receptor and induces apoptosis. Embo. J. 14: 4662-4675.

27. Kageyama R, Pastan I. (1989) Molecular cloning and characterization of a human DNA binding factor that represses transcription. Cell 59: 815-25.

28. Johnson AC, Kageyama R, Popescu NC, Pastan I. (1992) Expression and chromosomal localization of the gene for the human transcriptional repressor GCF. J. Biol. Chem. 267: 1689-1694.

29. Beguinot L, Yamazaki H, Pastan I, Johnson AC. (1995) Biochemical characterization of human GCF transcription factor in tumor cells. Cell Growth Differ. 6: 699-706.

30. Mu ZM, et al. (1994) PML, a growth suppressor disrupted in acute promyelocytic leukemia. Mol. Cell Biol. 14: 6858-6867.

31. Vallian S, et al. (1997) Transcriptional repression by the promyelocytic leukemia protein, PML. Exp. Cell Res. 237: 371-382.

32. Vallian S, et al. (1998) The promyelocytic leukemia protein interacts with $\mathrm{Spl}$ and inhibits its transactivation of the epidermal growth factor receptor promoter. Mol. Cell Biol. 18: 7147-7156.

33. de The H, et al. (1991) The PML-RAR alpha fusion mRNA generated by the $t(15 ; 17)$ translocation in acute promyelocytic leukemia encodes a functionally altered RAR. Cell 66: 675-684.

34. Chang KS, et al. (1992) Characterization of a fusion cDNA (RARA/myl) transcribed from the $t(15 ; 17)$ translocation breakpoint in acute promyelocytic leukemia. Mol. Cell Biol. 12: 800810.

35. Angel P, Karin M. (1991) The role of Jun, Fos and the AP-1 complex in cell-proliferation and transformation. Biochim. Biophys. Acta. 1072: 129-57.

36. Bohmann D, et al. (1987) Human proto-oncogene c-jun encodes a DNA binding protein with structural and functional properties of transcription factor AP-1. Science 238: 1386-1392.

37. Ryder K, et al. (1988) A gene activated by growth factors is related to the oncogene $\mathrm{v}$-jun. Proc. Natl. Acad. Sci. U.S.A. 85: 1487-1491.

38. Ryder K, et al. (1989) jun-D: a third member of the jun gene family. Proc. Natl. Acad. Sci. U.S.A. 86: 1500-1503.
39. Hirai SI, et al. (1989) Characterization of junD: a new member of the jun proto-oncogene family. Embo. J. 8: 1433-1439.

40. Miller AD, et al. (1984) c-fos protein can induce cellular transformation: a novel mechanism of activation of a cellular oncogene. Cell 36: 51-60.

41. Cohen DR, et al. (1988) fra-1: a seruminducible, cellular immediate-early gene that encodes a fos-related antigen. Mol. Cell. Biol. 8: 2063-2069.

42. Nishina $H$, et al. (1990) Isolation and characterization of fra-2, an additional member of the fos gene family. Proc. Natl. Acad. Sci. U.S.A. 87: 36193623.

43. Zerial $M$, et al. The product of a novel growth factor activated gene, fos B, interacts with JUN proteins enhancing their DNA binding activity. Embo. J. 8: 805-813.

44. Sassone-Corsi P, et al. (1990) Cross-talk in signal transduction: TPA-inducible factor jun/AP-1 activates cAMP-responsive enhancer elements. Oncogene 5: 427-431.

45. Hai T, et al. (1991) Cross-family dimerization of transcription factors Fos/Jun and ATF/CREB alters DNA binding specificity. Proc. Natl. Acad. Sci. U.S.A. 88: 3720-3724.

46. Smith LM, et al. (1997) Breast cancer cells have lower activating protein 1 transcription factor activity than normal mammary epithelial cells. Cancer Res. 57: 3046-3054.

47. Dynan WS, Tjian R. (1983) The promoterspecific transcription factor Spl binds to upstream sequences in the SV40 early promoter. Cell 35: 79-87.

48. Krylov D, et al. (1997) A general method to design dominant negatives to B-HLHZip proteins that abolish DNA binding. Proc. Natl. Acad. Sci. U.S.A. 94: 12274-12279.

49. Li Q, et al. (1998) Cisplatin induction of ERCC1 mRNA expression in A2780/CP70 human ovarian cancer cells. J. Biol. Chem. 273: 2341923425.

50. Li Q, et al. (1999) Modulation of excision repair cross complementation group 1 (ERCC-1) mRNA expression by pharmacological agents in human ovarian carcinoma cells. Biochem. Pharmacol. 57: 347-353.

51. Yang L, et al. (1997) Induction of retinoid resistance in breast cancer cells by overexpression of cJun. Cancer Res. 57: 4652-4661.

52. Johnson AC., Jinno Y, Merlino GT. (1988) Modulation of epidermal growth factor receptor proto-oncogene transcription by a promoter site sensitive to S1 nuclease. Mol. Cell Biol. 8: 41744184.

53. Rubinstein YR, et al. (1998) Interferon regulatory factor-1 is a major regulator of epidermal growth factor receptor gene expression. FEBS Lett. 431: 268-272. 
54. Hudson LG, Santon JB, Gill GN. (1989) Regulation of epidermal growth factor receptor gene expression. Mol. Endocrinol. 3: 400-408.

55. Karin M, Liu Z-g, Zandi E. (1997) AP- 1 function and regulation. Curr. Opin. Cell Biol. 9: 240-246.

56. Chen LL, Clawson ML, Bilgrami S, Carmichael G. (1993) A sequence-specific singlestranded DNA-binding protein that is responsive to epidermal growth factor recognizes an S1 nuclease-sensitive region in the epidermal growth factor receptor promoter. Cell Growth Differ. 4: 975-983.
57. Hou X, et al. (1994) Identification of an epidermal growth factor receptor transcriptional repressor. J. Biol. Chem. 269: 4307-4312.

58. Reed AL, et al. (1998) Molecular cloning and characterization of a transcription regulator with homology to GC-binding factor. J. Biol. Chem. 273: 21594-21602.

59. Hudson LG, Thompson KL, Xu J, Gill GN, Santon JB, Glass CK. (1990) Identification and characterization of a regulated promoter element in the epidermal growth factor receptor gene. Proc. Natl. Acad. Sci. U.S.A. 87: 7536-40. 